\title{
Confirmation and Control of HPPD-Inhibiting Herbicide-Resistant Waterhemp (Amaranthus tuberculatus) in Nebraska
}

\author{
Maxwel C. Oliveira, Amit J. Jhala, Todd Gaines, Suat Irmak, Keenan Amundsen, Jon E. Scott, and \\ Stevan Z. Knezevic*
}

Field and greenhouse experiments were conducted in Nebraska to (1) confirm the 4-hydroxyphenylpyruvate dioxygenase (HPPD)-inhibiting resistant-waterhemp biotype (HPPD-RW) by quantifying the resistance levels in dose-response studies, and (2) to evaluate efficacy of PRE-only, POST-only, and PRE followed by POST herbicide programs for control of HPPD-RW in corn. Greenhouse dose-response studies confirmed that the suspected waterhemp biotype in Nebraska has evolved resistance to HPPD-inhibiting herbicides with a 2- to 18-fold resistance depending upon the type of HPPD-inhibiting herbicide being sprayed. Under field conditions, at $56 \mathrm{~d}$ after treatment, $\geq 90 \%$ control of the HPPD-RW was achieved with PRE-applied mesotrione/atrazine/ $S$-metolachlor + acetochlor, pyroxasulfone (180 and $270 \mathrm{~g}^{\mathrm{ai} \mathrm{ha}} \mathrm{ha}^{-1}$ ), pyroxasulfone/fluthiacet-methyl/ atrazine, and pyroxasulfone + saflufenacil + atrazine. Among POST-only herbicide programs, glyphosate, a premix of mesotrione/atrazine tank-mixed with diflufenzopyr/dicamba, or metribuzin, or glufosinate provided $\geq 92 \%$ HPPD-RW control. Herbicide combinations of different effective sites of action in mixtures provided $\geq 86 \%$ HPPD-RW control in PRE followed by POST herbicide programs. It is concluded that the suspected waterhemp biotype is resistant to HPPD-inhibiting herbicides and alternative herbicide programs are available for effective control in corn. The occurrence of HPPD-RW in Nebraska is significant because it limits the effectiveness of HPPD-inhibiting herbicides.

Nomenclature: Acetochlor, atrazine, glyphosate, clopyralid, dicamba, diflufenzopyr, dimethenamid- $P$, flumetsulam, fluthiacet-methyl, glufosinate, isoxaflutole, mesotrione, metribuzin, pyroxasulfone $S$-metolachlor, saflufenacil, rimsulfuron, tembotrione, thiencarbazone-methyl, topramezone, waterhemp, Amaranthus tuberculatus (Moq.) Sauer, corn, Zea mays L.

Key words: 4-hydroxyphenylpyruvate dioxygenase, pigment inhibitors, PRE, POST, triketone, weed management, weed resistance.

\begin{abstract}
Se realizaron experimentos de campo y de invernadero en Nebraska para (1) confirmar un biotipo de Amaranthus tuberculatus resistente a inhibidores de 4-hydroxyphenylpyruvate dioxygenase (HPPD) (HPPD-RW) cuantificando el nivel de resistencia con estudios de respuesta a dosis, y (2) evaluar la eficacia de programas de herbicidas para el control de HPPD-RW en maíz con sólo herbicidas PRE, sólo POST, y herbicidas PRE seguidos por POST. Los estudios de respuesta a dosis en invernadero confirmaron que el biotipo de $A$. tuberculatus en Nebraska ha evolucionado resistencia a herbicidas inhibidores de HPPD con 2 a 18 veces mayor resistencia dependiendo del tipo de herbicida inhibidor de HPPD que se aplicó. Bajo condiciones de campo, a 56 d después del tratamiento, se alcanzó $\geq 90 \%$ de control de HPPD RW con aplicaciones PRE de mesotrione/ atrazine/S-metolachlor + acetochlor, pyroxasulfone (180 y $270 \mathrm{~g}$ ai $\left.\mathrm{ha}^{-1}\right)$, pyroxasulfone/fluthiacet-methyl/atrazine, y pyroxasulfone + saflufenacil + atrazine. Entre los programas de herbicidas con sólo POST, glyphosate, una premezcla de mesotrione/ atrazine mezclados en tanque con diflufenzopyr/dicamba, o metribuzin, o glufosinate brindaron $\geq 92 \%$ control de HPPD-RW. Combinaciones de herbicidas efectivos con diferentes sitios de acción en mezclas brindaron $\geq 86 \%$ de control de HPPD-RW en programas de herbicidas PRE seguidos por POST. Se concluyó que el biotipo de $A$. tuberculatus es resistente a herbicidas inhibidores de HPPD y que hay programas de herbicidas alternativos disponibles para su control efectivo en maíz. La ocurrencia de HPPD-RW en Nebraska es significativa porque limita la efectividad de herbicidas inhibidores de HPPD.
\end{abstract}

\footnotetext{
DOI: $10.1017 /$ wet.2016.4

${ }^{*}$ First, second, and fifth authors: Graduate Student, Assistant Professor, and Assistant Professor, Department of Agronomy and Horticulture, University of Nebraska, Lincoln, NE 68583; Third author: Assistant Professor, Department of Bioagricultural Sciences and Pest Management, Colorado State University, Fort Collins, CO 80523; Fourth author: Professor, Department of Biological Systems Engineering, University of Nebraska, Lincoln, NE 68583; Sixth and seventh authors: Research Technologist and Professor, Northeast Research and Extension Center, Haskell Agricultural Laboratory, University of Nebraska-Lincoln, Concord, NE 68728. Corresponding author's E-mail: sknezevic2@unl.edu
} 
Waterhemp is a summer, annual, broadleaf species native to the midwestern United States (Sauer 1967; Waselkov and Olsen 2014). Waterhemp has been identified as one of the most troublesome weeds over the past decade (Hager et al. 2002; Prince et al. 2012; Steckel and Sprague 2004). There are a variety of factors contributing to the rise of waterhemp as a problem weed, including adoption of no-tillage farming practices, extended germination period of waterhemp, decreased use of residual herbicides, and rapid spread of multiple herbicide resistance (Culpepper 2006; Felix and Owen 1999; Hartzler et al. 1999).

Waterhemp biotypes have evolved resistance to six herbicide site-of-action (SOA) groups, including acetolactate synthase inhibitors (Weed Science Society of America Site of Action [WSSA SAO] Group 2), synthetic auxins (WSSA SOA Group 4), triazines (WSSA SOA Group 5), 5-enolpyruvylshikimate3-phosphate synthase inhibitors (WSSA SOA Group 9), and protoporphyrinogen oxidase inhibitors (WSSA SOA Group 14) (Bernards et al. 2012; Heap 2016a; Sarangi et al. 2015; Tranel et al. 2011). In addition, just in the last six years it was confirmed that waterhemp biotypes evolved resistance to 4-hydroxyphenylpyruvate dioxygenase (HPPD)-inhibiting herbicides (WSSA SOA Group 27) in Illinois (Hausman et al. 2011) and Iowa (McMullan and Green 2011). Therefore, new herbicide options to manage waterhemp are needed (Tranel et al. 2011). However, no new herbicide SOAs have been developed in recent years (Duke 2012).

The herbicides with the most recently developed SOA are the HPPD-inhibiting herbicides, introduced in the 1980s (Duke 2012; Mitchell et al. 2001). This herbicide group inhibits HPPD and causes bleaching of green tissues of susceptible plants (Grossmann and Ehrhardt 2007; Mitchell et al. 2001). Mesotrione, tembotrione, and topramezone are common POST-applied HPPD-inhibiting herbicides, primarily used in corn (Bollman et al. 2008; Gitsopoulos et al. 2010; Nurse et al. 2010; Sutton et al. 2002). The recent occurrence of a HPPD-inhibiting herbicide-resistant waterhemp biotype (HPPD-RW) has increased the complexity of waterhemp control in corn (McMullan and Green 2011).

Control of a waterhemp biotype in Illinois that is resistant to HPPD- and photosystem II-inhibiting herbicides was not achieved using a single active ingredient of foliar or soil-applied herbicide (Hausman et al. 2015; Hausman et al. 2013); therefore, there is a need for including the use of mixtures in herbicide programs to control HPPD-RW. The benefits of using herbicide mixtures are well documented, including season-long weed control and a reduction in the risk of herbicide resistance (Beckie and Reboud 2009; Butts et al. 2016; Johnson et al. 2012; Kumar and Jha 2015; Loux et al. 2011).

Failure of a POST-applied HPPD-inhibiting herbicide to control waterhemp in a seed corn production operation was reported in eastern Nebraska in 2011. Therefore, we conducted a series of experiments to 1) confirm the presence of HPPD-RW and determine its level of resistance to POST-applied mesotrione, tembotrione, and topramezone in dose-response studies, and to 2) evaluate herbicide options for control of HPPD-RW based on PRE-only, POSTonly, and PRE followed by (fb) POST herbicide programs. This information will be beneficial in the development of alternative herbicide programs for managing HPPD-RW in Nebraska.

\section{Materials and Methods}

Plant Materials. In the fall of 2013, inflorescences of waterhemp plants that survived repeated mesotrione and tembotrione applications were collected from a field near Columbus, Platte County, NE, and used as the suspected HPPD-RW. Waterhemp inflorescences collected in the fall of 2014 from a field in Clay County, NE with a history of effective control using the recommended rate of HPPDinhibiting herbicides were considered the HPPDinhibiting herbicide-susceptible waterhemp biotype (HPPD-SW), and used in this study for a comparison. Inflorescences of waterhemp were dried for $2 \mathrm{wk}$ at room temperature $(25 \mathrm{C})$. The seeds were cleaned and stored at $5 \mathrm{C}$ until used in the greenhouse study. Seeds were planted in $713-\mathrm{cm}^{3}$ plastic pots containing a commercial potting mix (Berger BM1 All-Purpose Mix, Berger Peat Moss Ltd., Saint-Modest, Quebec, Canada). Emerged seedlings $(1 \mathrm{~cm})$ were transplanted into $164-\mathrm{cm}^{3}$ cone-tainers (Ray Leach "Cone-tainer" SC10®, Stuewe and Sons Inc, Tangent, OR 97389) containing identical commercial potting mix described above. Plants were supplied with adequate water and kept in greenhouse conditions at 28/22 C day/night temperature. Artificial lighting was provided using metal halide lamps $\left(600 \mu \mathrm{mol}\right.$ photon $\left.\mathrm{m}^{-2} \mathrm{~s}^{-1}\right)$ to ensure a $16-\mathrm{h}$ photoperiod. 
Dose-Response Studies. Greenhouse dose-response bioassays were conducted in 2015 at the University of Nebraska-Lincoln to determine the resistance levels of HPPD-RW and HPPD-SW sprayed with each of the three HPPD-inhibiting herbicides (mesotrione, tembotrione, and topramezone).

Each study had a completely randomized design with four replications and was repeated twice. Separate experiments were conducted for the HPPD-RW and the HPPD-SW. The treatments were arranged in a factorial treatment design with 3 herbicides and 6 rates. The herbicide rates for the HPPD-RW were $0,0.5 \times, 1 \times$, $2 x, 4 x$, and $8 x$, and for the HPPD-SW were $0,0.25 \times$, $0.5 \times, 0.75 \times, 1 \times$, and $2 \times$, where $1 \times$ represents either $105 \mathrm{~g}$ ai ha ${ }^{-1}$ mesotrione (Syngenta Crop Protection, Research Triangle Park, NC 27709) plus 1\% v/v of crop oil concentrate (Agri-Dex®, Helena Chemical Co., Collierville, TN 38017) and $20.5 \mathrm{~g} \mathrm{~L}^{-1}$ of ammonium sulfate (DSM Chemicals North America Inc., Augusta, GA 30901); $92 \mathrm{~g}$ ai ha ${ }^{-1}$ tembotrione (Bayer Crop Science, Research Triangle Park, NC 27709) plus 1\% v/ $\mathrm{v}$ methylated seed oil (Noble®, Winfield Solutions, Shoreview, MN 55126) and $20.5 \mathrm{~g} \mathrm{~L}^{-1}$ ammonium sulfate; or $24.5 \mathrm{~g}$ ai ha ${ }^{-1}$ topramezone (AMVAC, Los Angeles, CA 90023) plus 1\% v/v methylated seed oil and $20.5 \mathrm{~g} \mathrm{~L}^{-1}$ ammonium sulfate.

Herbicide treatments were applied with a singletip chamber sprayer (DeVries Manufacturing Corp, Hollandale, MN 56045) fitted with an 8001 E nozzle (Spraying Systems Co., North Avenue, Wheaton, IL 60139), calibrated to deliver $140 \mathrm{~L} \mathrm{ha}^{-1}$ spray volume at $210 \mathrm{kPa}$ at a speed of $3.7 \mathrm{~km} \mathrm{~h}^{-1}$. Waterhemp control was assessed visually $21 \mathrm{~d}$ after treatment (DAT) using a scale of $0 \%$ to $100 \%$ (where 0 indicates no injury and 100 indicates plant death). Control ratings were based on symptoms such as bleaching, necrosis, and stunting of plants compared to non-treated plants. Aboveground biomass was harvested at 21 DAT from each experimental unit and oven-dried at $65 \mathrm{C}$ until reaching constant weight before weight of biomass was recorded. The biomass $(\mathrm{g})$ data were converted into biomass reduction (\%) compared with the non-treated experimental unit as:

\% HPPD-RW biomass reduction

$$
=[(\bar{E}-B) / \bar{E}] \times 100,
$$

where $\bar{E}$ represents the mean biomass (g) of the non-treated experimental unit replicates, and
$B$ represents the biomass ( $\mathrm{g}$ ) of an individual treated experimental unit.

The effective dose needed to suppress the population by $50 \%\left(\mathrm{ED}_{50}\right)$ and $90 \%\left(\mathrm{ED}_{90}\right)$ for HPPD-RW and HPPD-SW was determined using the three-parameter log-logistic curve of the drc package of the $\mathrm{R}$ statistical environment (Knezevic et al. 2007):

$$
Y=d / 1+\exp \{b[\log (x)-\log (e)]\} .
$$

In this model, $Y$ is the control (\%) or biomass reduction $(\%), d$ is the upper limit, and $e$ represents the $\mathrm{ED}_{50}$ value. The parameter $b$ is the relative slope around the parameter $e$, and $x$ is the herbicide dose in $\mathrm{g}$ ai ha ${ }^{-1}$.

The resistance level was calculated by dividing the effective dose $\left(E_{50}\right)$ of the HPPD-RW by the effective dose of the HPPD-SW. The resistance level indices for the respective effective dose between the HPPD-RW and the HPPD-SW were compared using the EDcomp (or SI) function of package drc in $\mathrm{R}$ software (Ritz and Streibig 2005). The EDcomp function compares the ratio of effective doses using $t$-statistics, where $P$-value $<0.05$ indicates that herbicide $\mathrm{ED}_{50}$ values are different between the HPPD-RW and the HPPD-SW. The Fligner-Killeen test of homogeneity was used to test the assumption of constant error variance among data sets. This is a non-parametric test, which can detect departures from normality in data (Conover et al. 1981).

Efficacy of Herbicide Programs on HPPDRW. Field experiments were conducted in 2013 and 2014 at a Platte County field location near Columbus, $\mathrm{NE}\left(41.64^{\circ} \mathrm{N}, 97.58^{\circ} \mathrm{W}\right)$ where the HPPD-RW was reported. The soil type at the study location was a silty clay loam ( $12 \%$ sand, $60 \%$ silt, $28 \%$ clay) with $3.3 \%$ organic matter and a $\mathrm{pH}$ of 6.8 . Glyphosate- and glufosinate-tolerant hybrid corn 'Golden Harvest H-9138' was seeded at 79,280 seeds $\mathrm{ha}^{-1}$ in rows spaced $76 \mathrm{~cm}$ apart on May 16, 2013 and May 22, 2014. Monthly mean air temperature and total precipitation data during the study periods are provided (Table 1). Experiments were conducted in a randomized complete block design with three replications and 10, 6, and 16 treatments for PRE-only, POST-only, and PRE fb POST herbicide programs, respectively (Tables 2,3 , and 4 ). A 3 by $7.6 \mathrm{~m}$ plot was considered an experimental unit.

Herbicide treatments were applied with a $\mathrm{CO}_{2}$ pressurized backpack sprayer calibrated to deliver $140 \mathrm{~L} \mathrm{ha}^{-1}$ aqueous solution at $172 \mathrm{kPa}$ (PRE) and 
Table 1. Mean monthly air temperature and total precipitation in field experiments conducted in 2013 and 2014 near Columbus, NE.

\begin{tabular}{|c|c|c|c|c|c|c|}
\hline \multirow[b]{2}{*}{ Month $^{\mathrm{a}}$} & \multicolumn{3}{|c|}{ Temperature } & \multicolumn{3}{|c|}{ Total precipitation } \\
\hline & 2013 & 2014 & 50-y avg. & 2013 & 2014 & 50-y avg. \\
\hline & & $-c$ & & & $-\mathrm{mn}$ & \\
\hline May & 15 & 16 & 17 & 83 & 96 & 112 \\
\hline June & 21 & 21 & 22 & 120 & 200 & 118 \\
\hline July & 23 & 22 & 25 & 29 & 108 & 81 \\
\hline
\end{tabular}

a Abbreviations: Weather data were obtained from the High Plains Regional Climate Center (HPRCC; http://www.hprcc.unl.edu).

$240 \mathrm{kPa}$ (POST) with a $2 \mathrm{~m}$ spray boom through Turbo Teejet ${ }^{\circledR} 11002$ (PRE) and 110015 (POST) flat fan sprayer nozzles at a speed of $4.3 \mathrm{~km} \mathrm{~h}^{-1}$. The PRE herbicides were applied on May 17, 2013 and May 23, 2014, and the POST herbicides were applied when the HPPD-RW was 8 to $10 \mathrm{~cm}$ tall. The HPPD-RW control was visually assessed at 30, 41, and 56 DAT (PRE-only); 7, 14, and 21 DAT (POST-only); and $30 \mathrm{~d}$ after PRE (DAPRE), and 32 $\mathrm{d}$ after POST (DAPOST) (PRE fb POST) on a scale ranging from $0 \%$, indicating no control, to $100 \%$, indicating complete control. HPPD-RW density was determined at 56 DAT (PRE), 35 DAT (POST), and 32 DAPOST (PRE fb POST) by counting waterhemp within $0.25 \mathrm{~m}^{2}$ quadrats arbitrarily placed between the middle two corn rows in each experimental unit. The HPPD-RW densities in the non-treated experimental unit averaged of 196 and 344 plants $\mathrm{m}^{-2}$ in 2013 and 2014 , respectively. The HPPD-RW density (plants $\mathrm{m}^{-2}$ ) data were expressed as HPPD-RW density reduction (\%) and compared with the non-treated experimental unit as follows:

\% HPPD-RW density reduction

$$
=[(\dot{C}-D) / \dot{C}] \times 100
$$

Where $C$ is the mean HPPD-RW density (plants $\mathrm{m}^{-2}$ ) of the non-treated experimental unit replicates, and $D$ is the HPPD-RW density (plants $\mathrm{m}^{-2}$ ) of an individual treated experimental unit.

ANOVA was performed using PROC GLIMMIX in SAS version 9.3 (SAS Institute Inc., Cary, NC 27513). Fligner-Killeen tests of homogeneity of variances between years and treatment-by-year interactions were conducted. HPPD-RW control (\%) and density reduction (\%) were analyzed with beta distribution with ilink function to meet assumptions of residual variance analysis. If ANOVA indicated significant treatment effects, means were separated at $P \leq 0.05$ with Fisher's protected LSD test.

Table 2. List of PRE-only herbicides used for control of HPPD-inhibiting herbicide-resistant waterhemp in field experiments conducted in 2013 and 2014 near Columbus, NE.

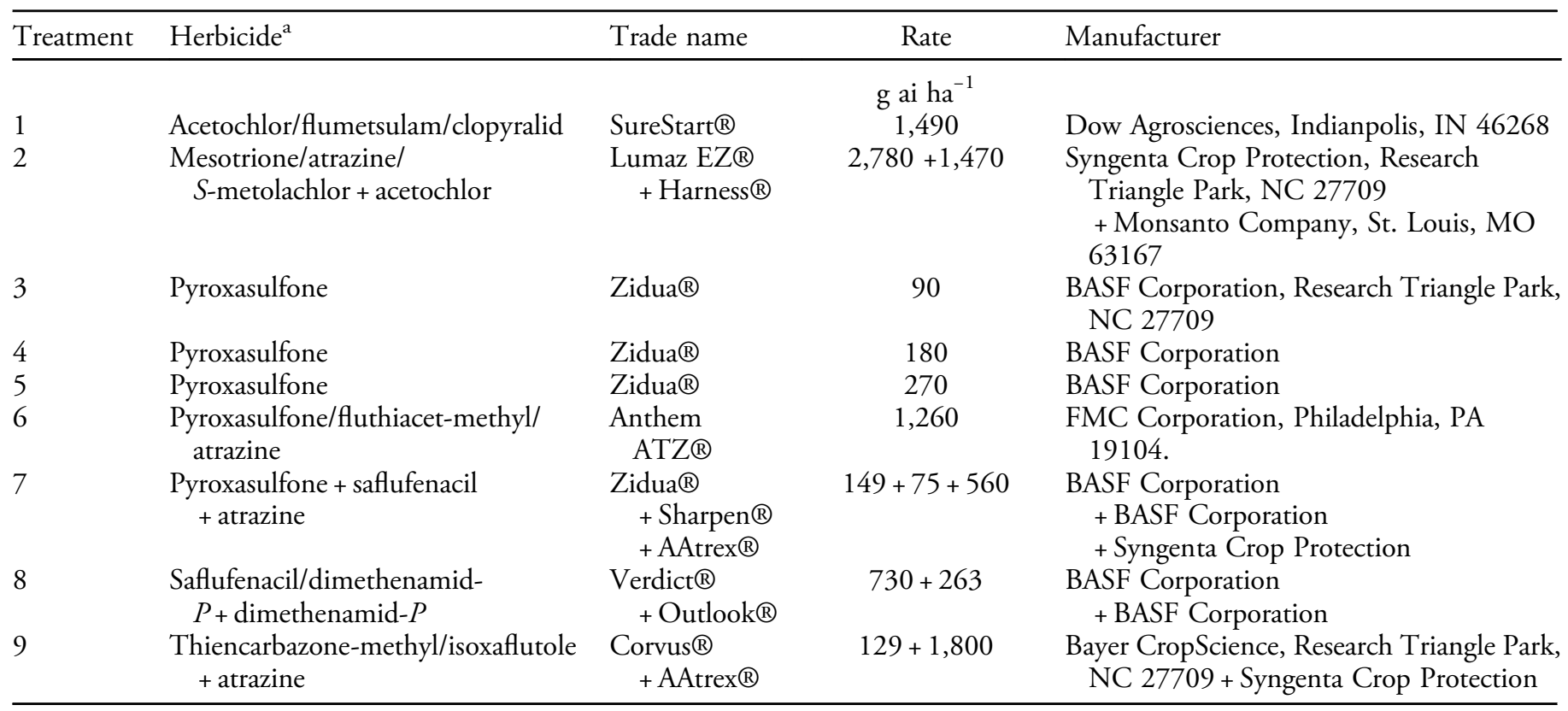

a Abbreviations: Herbicide premix (/); herbicide tankmix (+). 
Table 3. List of POST-only herbicides used for control of HPPD-inhibiting herbicide-resistant waterhemp in field experiments conducted in 2013 and 2014 near Columbus, NE.

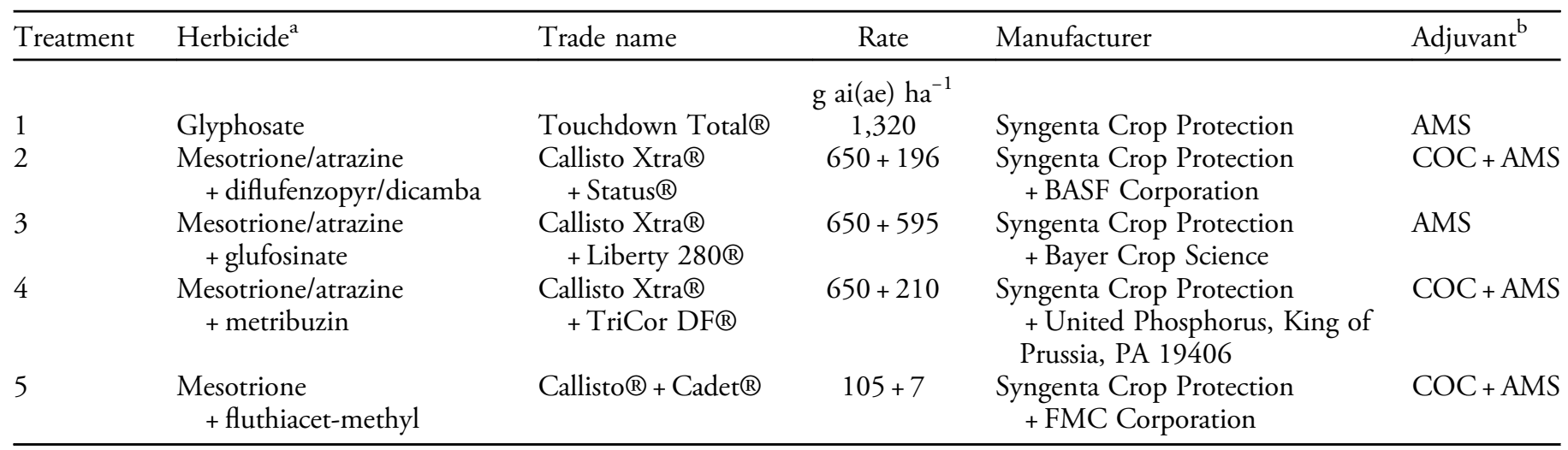

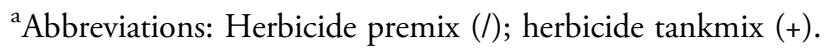

${ }^{\mathrm{b}}$ AMS, ammonium sulfate (20.5 $\mathrm{g} \mathrm{L}^{-1}$; DSM Chemicals North America Inc., Augusta, GA 30901); COC, crop oil concentrate (1\% v/v; Agridex, Helena Chemical Co., Collierville,TN 38017).
}

\section{Results and Discussion}

Dose-Response Studies. Error variance among data sets was constant. Treatment-by-experiment interaction was not significant; therefore data were combined.

Dose-response studies confirmed that the waterhemp biotype was resistant to POST-applied HPPD-inhibiting herbicides (mesotrione, tembotrione, and topramezone). The labeled rate of mesotrione $\left(105 \mathrm{~g} \mathrm{ha}^{-1}\right)$ provided less than $40 \%$ control of the HPPD-RW (Figure 1A). In addition, a mesotrione rate of $342 \mathrm{~g} \mathrm{ha}^{-1}$ was needed to achieve $50 \%\left(\mathrm{ED}_{50}\right)$ control of the HPPD-RW (Table 5), which is thirteen times the rate required to control HPPD-SW. The $\mathrm{ED}_{90}$ was not calculated because $90 \%$ control was not achieved even with the maximum rate $\left(840 \mathrm{~g} \mathrm{ha}^{-1}\right)$ of mesotrione tested in this study. A similar trend was evident with tembotrione (Figure $2 \mathrm{~A}$ ) and topramezone (Figure 3A), with the resistance level, based on $\mathrm{ED}_{50}$ values, estimated as 6-and 3-fold higher for HPPD-RW than for HPPD-SW, respectively. In contrast, the HPPD-SW demonstrated sensitivity to all three HPPD-inhibiting herbicides applied POST: 90\% control $\left(\mathrm{ED}_{90}\right)$ was achieved with the labeled rates (Table 5).

Dose-response curves based on biomass reduction (\%) suggest the same resistance level as do the data based on control (\%) estimates (Figures. 1B, 2B, and $3 \mathrm{~B}$; Table 6 ). Based on biomass reduction (\%), the HPPD-SW was most resistant to mesotrione (18-fold), followed by tembotrione (5-fold), and topramezone (2-fold). Higher resistance levels to mesotrione are likely due to longer use history of mesotrione-based products for weed control at the research site.

McMullan and Green (2011) reported waterhemp resistant to mesotrione in Iowa, but at a lower level of resistance (8-fold). Differences in fold-level resistance between reported biotypes may partly be due to variation in the sensitivity of the susceptible population used in the study, and may also be due to the fact that the waterhemp biotype from Iowa was also acetolactate synthase- and triazine-resistant. In addition, HPPD herbicide resistance level may be influenced by plant height at the time of application. The Iowa waterhemp biotype height at the time of application was 3 to $5 \mathrm{~cm}$, compared to 8 to $10 \mathrm{~cm}$ in this study. Furthermore, multiple resistant populations of waterhemp in Illinois also exhibited various resistance levels to mesotrione, ranging 10- to 35-fold, depending upon the susceptible population used for comparison (Hausman et al. 2011). Palmer amaranth resistant to HPPD-inhibiting herbicides has also been confirmed in Nebraska (Jhala et al. 2014) and Kansas (Thompson et al. 2012). In Nebraska, it was reported that Palmer amaranth sprayed when $10 \mathrm{~cm}$ tall was most resistant to topramezone (14to 23-fold), followed by tembotrione (4- to 6-fold), and mesotrione (4-fold) (Jhala et al. 2014).

Efficacy of Herbicide Programs on HPPDRW. Error variance among data sets was constant. Treatment-by-year interaction was not significant for the three field experiments; therefore, data were combined.

Oliveira et al.: HPPD Resistant Waterhemp • 71 
Table 4. List of PRE followed by POST herbicides used for control of HPPD-inhibiting herbicide-resistant waterhemp in field experiments conducted in 2013 and 2014 near Columbus, Platte County, NE.

\begin{tabular}{|c|c|c|c|c|c|c|}
\hline Treatment & Herbicide $^{\mathrm{a}}$ & Trade name & Timing & Rate & Manufacturer & Adjuvant $^{\mathrm{b}}$ \\
\hline 1 & $\begin{array}{l}\text { Acetochlor/atrazine } \mathrm{fb} \\
\text { glyphosate } \\
\quad \text { + topramezone + atrazine }\end{array}$ & $\begin{array}{l}\text { Harness Xtra® fb } \\
\text { Roundup PowerMax® } \\
\quad+\text { Impact® + AAtrex® }\end{array}$ & $\begin{array}{l}\text { PRE fb } \\
\text { POST }\end{array}$ & $\begin{array}{l}\mathrm{g} \text { ai(ae) ha }{ }^{-1} \\
2,700 \mathrm{fb} \\
+1,540+25+560\end{array}$ & $\begin{array}{l}\text { Monsanto Company } \mathrm{fb} \\
\text { Monsanto Company } \\
\text { + AMVAC, Commerce, CA } 90040 \\
\text { + Syngenta Crop Protection }\end{array}$ & $\mathrm{MSO}+\mathrm{AMS}$ \\
\hline 2 & $\begin{array}{l}\text { Acetochlor/atrazine } \mathrm{fb} \\
\text { topramezone + diflufenzopyr/dicamba } \\
\quad+\text { atrazine }\end{array}$ & $\begin{array}{l}\text { Harness Xtra® fb } \\
\text { Impact }{ }^{\circledR}+\text { Status }{ }^{\circledR}+\text { AAtrex® }\end{array}$ & $\begin{array}{l}\text { PRE fb } \\
\text { POST }\end{array}$ & $\begin{array}{c}2,700 \mathrm{fb} \\
25+196+560\end{array}$ & $\begin{array}{l}\text { Monsanto Company fb } \\
\text { + AMVAC + BASF Corporation } \\
\text { + Syngenta Crop Protection }\end{array}$ & $\mathrm{MSO}+\mathrm{AMS}$ \\
\hline 3 & $\begin{array}{l}\text { Acetochlor/flumetsulam/clopyralid fb } \\
\text { glyphosate }\end{array}$ & $\begin{array}{l}\text { SureStart }{ }^{\circledR} \mathrm{fb} \\
\text { Durango }{ }^{\circledR}\end{array}$ & $\begin{array}{l}\text { PRE fb } \\
\text { POST }\end{array}$ & $\begin{array}{c}1,490 \mathrm{fb} \\
1,170\end{array}$ & $\begin{array}{l}\text { Dow Agrosciences } \mathrm{fb} \\
\text { Dow Agrosciences }\end{array}$ & AMS \\
\hline 4 & $\begin{array}{l}\text { Mesotrione/atrazine/S-metolachlor } \\
\quad+\text { atrazine + acetochlor } \mathrm{fb}\end{array}$ & $\begin{array}{l}\text { Lumaz EZ® + AAtrex® } \\
+ \text { Harness }{ }^{\circledR} \mathrm{fb}\end{array}$ & PRE fb & $\begin{array}{c}2,780+1,080+1,470 \\
\mathrm{fb}\end{array}$ & $\begin{array}{l}\text { Syngenta Crop Protection }+ \text { Syngenta } \\
\text { Crop Protection + Monsanto Company } \\
\mathrm{fb}\end{array}$ & \\
\hline & diflufenzopyr/dicamba + atrazine & Status ${ }^{\circledR}+$ AAtrex ${ }^{\circledR}$ & POST & $196+450$ & $\begin{array}{l}\text { BASF Corporation + Syngenta Crop } \\
\text { Protection }\end{array}$ & $\begin{array}{l}\text { COC } \\
+ \text { AMS }\end{array}$ \\
\hline 5 & $\begin{array}{l}\text { Mesotrione/atrazine/S-metolachlor } \\
+ \text { atrazine } \mathrm{fb}\end{array}$ & Lumaz EZ® + AAtrex® fb & PRE fb & $2,780+1,080 \mathrm{fb}$ & $\begin{array}{l}\text { Syngenta Crop Protection } \\
\quad+\text { Syngenta Crop Protection } \mathrm{fb}\end{array}$ & \\
\hline & diflufenzopyr/dicamba + glyphosate & Status® + Touchdown Total® & POST & $196+1170$ & $\begin{array}{l}\text { BASF Corporation } \\
\quad+\text { Syngenta Crop Protection }\end{array}$ & AMS \\
\hline 6 & $\begin{array}{l}\text { Mesotrione/atrazine/ } S \text {-metolachlor } \\
+ \text { atrazine } \mathrm{fb}\end{array}$ & Lumaz EZ® + AAtrex® fb & PRE $\mathrm{fb}$ & $2,780+1,080 \mathrm{fb}$ & $\begin{array}{l}\text { Syngenta Crop Protection } \\
\text { + Syngenta Crop Protection } \mathrm{fb}\end{array}$ & \\
\hline & glyphosate & Touchdown Total $®$ & POST & 1,170 & Syngenta Crop Protection & AMS \\
\hline 7 & $\begin{array}{l}\text { Mesotrione/atrazine } / S \text {-metolachlor } \\
\quad+\text { atrazine } \mathrm{fb} \\
\text { atrazine }+S \text {-metolachlor }+ \text { glufosinate }\end{array}$ & $\begin{array}{l}\text { Lumaz EZ® } \\
+ \text { AAtrex }{ }^{\circledR} \text { fb } \\
\text { AAtrex® + Dual II Magnum }{ }^{\circledR} \\
\quad+\text { Liberty } 280 \AA\end{array}$ & $\begin{array}{l}\text { PRE fb } \\
\text { POST }\end{array}$ & $\begin{array}{c}2,780 \\
+1,080 \mathrm{fb} \\
450+1,070+595\end{array}$ & $\begin{array}{l}\text { Syngenta Crop Protection } \\
\text { + Syngenta Crop Protection fb } \\
\text { Syngenta Crop Protection } \\
\text { + Syngenta Crop Protection } \\
\text { + Bayer Crop Science }\end{array}$ & AMS \\
\hline 8 & $\begin{array}{l}\text { Mesotrione/atrazine/S-metolachlor } \mathrm{fb} \\
\text { atrazine + glyphosate/ } S \text {-metolachlor/ } \\
\text { mesotrione }\end{array}$ & $\begin{array}{l}\text { Lumaz EZ® fb } \\
\text { AAtrex® }+ \text { Halex® GT }\end{array}$ & $\begin{array}{l}\text { PRE fb } \\
\text { POST }\end{array}$ & $\begin{array}{c}1,550 \mathrm{fb} \\
1,080+2,220\end{array}$ & $\begin{array}{l}\text { Syngenta Crop Protection fb } \\
\text { Syngenta Crop Protection } \\
\quad+\text { Syngenta Crop Protection }\end{array}$ & NIS + AMS \\
\hline 9 & $\begin{array}{l}\text { Mesotrione/atrazine/ } S \text {-metolachlor fb } \\
\text { glyphosate }\end{array}$ & $\begin{array}{l}\text { Lumaz EZ® fb } \\
\text { Touchdown Total }{ }^{\circ}\end{array}$ & $\begin{array}{l}\text { PRE fb } \\
\text { POST }\end{array}$ & $\begin{array}{c}2,780 \mathrm{fb} \\
1,170\end{array}$ & $\begin{array}{l}\text { Syngenta Crop Protection fb } \\
\text { Syngenta Crop Protection }\end{array}$ & AMS \\
\hline 10 & $\begin{array}{l}\text { Mesotrione/atrazine/S-metolachlor } \mathrm{fb} \\
\text { diflufenzopyr/dicamba }\end{array}$ & $\begin{array}{l}\text { Lumaz EZ® fb } \\
\text { Status® }\end{array}$ & $\begin{array}{l}\text { PRE fb } \\
\text { POST }\end{array}$ & & $\begin{array}{l}\text { Syngenta Crop Protection } \mathrm{fb} \\
\text { BASF Corporation }\end{array}$ & NIS \\
\hline 11 & $\begin{array}{l}\text { Mesotrione/atrazine/S-metolachlor fb } \\
\text { diflufenzopyr/dicamba }\end{array}$ & $\begin{array}{l}\text { Lumaz EZ® fb } \\
\text { Status }{ }^{\circledR}\end{array}$ & $\begin{array}{l}\text { PRE fb } \\
\text { POST }\end{array}$ & $\begin{array}{c}2,780 \mathrm{fb} \\
196\end{array}$ & $\begin{array}{l}\text { Syngenta Crop Protection } \mathrm{fb} \\
\text { BASF Corporation }\end{array}$ & NIS \\
\hline 12 & $\begin{array}{l}\text { Pyroxasulfone/fluthiacet-methyl/atrazine } \mathrm{fb} \\
\text { pyroxasulfone/fluthiacet-methyl }\end{array}$ & $\begin{array}{l}\text { Anthem ATZ® fb } \\
\text { Anthem }{ }^{8}\end{array}$ & $\begin{array}{l}\text { PRE fb } \\
\text { POST }\end{array}$ & $\begin{array}{c}1,260 \mathrm{fb} \\
94\end{array}$ & $\begin{array}{l}\text { FMC Corporation } \mathrm{fb} \\
\text { FMC Corporation }\end{array}$ & NIS \\
\hline 13 & Rimsulfuron $+S$-metolachlor/atrazine $\mathrm{fb}$ & $\begin{array}{l}\text { Resolve DF® + Bicep II Magnum }{ }^{\circledR} \\
\mathrm{fb}\end{array}$ & PRE fb & $18+3,240$ & $\begin{array}{l}\text { DuPont, Wilmington, DE } \\
19898 \text { + Syngenta Crop Protection fb }\end{array}$ & \\
\hline 14 & $\begin{array}{l}\text { glyphosate } \\
\text { Saflufenacil/dimethenamid- } P \\
\text { + dimethenamid- } P \text { fb }\end{array}$ & $\begin{array}{l}\text { Abundit Extra }{ }^{\circledR} \\
\text { Verdict }{ }^{\circledR}+\text { Outlook }{ }^{\circledR} \text { fb }\end{array}$ & $\begin{array}{l}\text { POST } \\
\text { PRE fb }\end{array}$ & $\begin{array}{c}840 \\
730+263 \mathrm{fb}\end{array}$ & $\begin{array}{l}\text { DuPont } \\
\text { BASF Corporation }\end{array}$ & AMS \\
\hline 15 & $\begin{array}{l}\text { topramezone + diflufenzopyr/dicamba } \\
\text { Thiencarbazone-methyl/isoxaflutole + } \\
\text { atrazine } \mathrm{fb} \\
\text { diflufenzopyr/dicamba }\end{array}$ & $\begin{array}{l}\text { Impact }{ }^{\circledR}+\text { Status }{ }^{\circledR} \\
\text { Corvus }{ }^{\circledR}+\text { AAtrex } ® \mathrm{fb} \\
\text { Status }{ }^{\circledR}\end{array}$ & $\begin{array}{l}\text { POST } \\
\text { PRE fb } \\
\text { POST }\end{array}$ & $\begin{array}{c}18+196 \\
129+1,120 \mathrm{fb} \\
196\end{array}$ & $\begin{array}{l}\text { + BASF Corporation fb } \\
\text { AMVAC + BASF Corporation } \\
\text { Bayer Crop Science + } \\
\text { Syngenta Crop Protection fb } \\
\text { BASF Corporation }\end{array}$ & NIS \\
\hline
\end{tabular}

${ }^{a}$ Abbreviations: Herbicide premix (/); herbicide tankmix (+); fb, followed by.

${ }^{\mathrm{b}}$ AMS, ammonium sulfate (20.5 g L ${ }^{-1}$; DSM Chemicals North America Inc., Augusta, GA 30901); COC, crop oil concentrate (1\% v/v; Agridex®, Helena Chemical Co., Collierville, TN 38017); NIS, nonionic surfactant (0.25\% v/v; Induce®, Helena Chemical Co., Collierville, TN 38017), MSO, methylated seed oil (1\% v/v; Noble@, Winfield Solutions, Shoreview, MN 55126). 


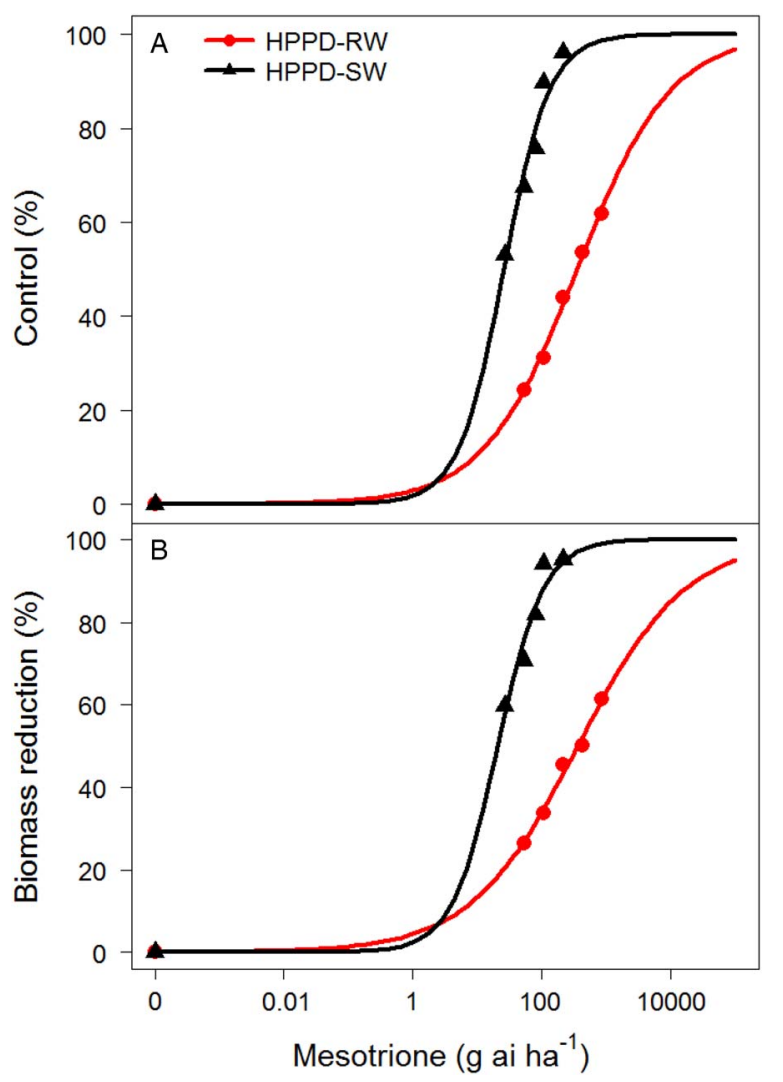

Figure 1. Control of (A) and biomass reduction (B) of 8- to 10-cm tall HPPD-inhibiting herbicide-resistant (HPPD-RW) and susceptible (HPPD-SW) waterhemp biotype at $21 \mathrm{~d}$ after treatment with POST-applied mesotrione in dose-response studies under greenhouse conditions.

PRE-Only Herbicide Program. PRE herbicides evaluated in this study provided $51 \%$ to $96 \%$ control and density reduction of the HPPD-RW (Table 7). Pyroxasulfone applied alone at $270 \mathrm{~g} \mathrm{ha}^{-1}$ and several other PRE-applied herbicide mixtures with different SOA (Treatments 1, 2, 6, 7, 8, and 9) provided $\geq 93 \%$ HPPD-RWcontrol at 30 and 41 DAT. At 56 DAT, mesotrione/atrazine/S-metolachlor, pyroxasulfone (180 and $\left.270 \mathrm{~g} \mathrm{~h}^{-1}\right)$, and pyroxasulfone + saflufenacil + atrazine provided $95 \%$ to $98 \%$ control without difference among them. Moreover, at 56 DAT, there was no difference between higher pyroxasulfone rates (180 and $270 \mathrm{~g} \mathrm{ha}^{-1}$ ) and pyroxasulfone applied in tank-mixtures with other herbicides (Treatments 6 and 7). Similarly, other studies have shown $\geq 90 \%$ control of pigweed species with pyroxasulfone applied alone or in tank-mixtures (Knezevic et al. 2009; Mahoney et al. 2014; Nurse et al. 2010).
Table 5. Estimated $\mathrm{ED}_{50}$ and $\mathrm{ED}_{90}$ values based on control (\%) in 8- to 10-cm HPPD-inhibiting herbicide-resistant (HPPD-R) and susceptible (HPPD-S) waterhemp biotype at $21 \mathrm{~d}$ after treatment in a dose-response study with mesotrione, tembotrione, and topramezone conducted under greenhouse conditions at the University of Nebraska-Lincoln.

\begin{tabular}{|c|c|c|c|c|}
\hline \multirow[b]{2}{*}{ Biotype $^{a}$} & \multicolumn{2}{|c|}{ HPPD-inhibiting herbicides } & \multirow[b]{2}{*}{$P$-value ${ }^{\mathrm{c}}$} & \multirow[b]{2}{*}{$\begin{array}{c}\text { Resistance } \\
\text { level }^{\mathrm{d}}\end{array}$} \\
\hline & $\begin{array}{l}\mathrm{ED}_{50} \\
( \pm \mathrm{SE})\end{array}$ & $\begin{array}{l}\mathrm{ED}_{90} \\
( \pm \mathrm{SE})\end{array}$ & & \\
\hline & \multicolumn{2}{|c|}{$\begin{array}{l}\text { g ai ha } \\
\text { Mesotrione }\end{array}$} & & \\
\hline HPPD-SW & $26(2)$ & $152(17)$ & & - \\
\hline HPPD-RW & $342(28)$ & - & $* * *$ & 13 \\
\hline \multicolumn{3}{|c|}{ Tembotrione } & & \\
\hline HPPD-SW & $11(2)$ & $57(6)$ & & - \\
\hline HPPD-RW & $61(4)$ & $673(99)$ & $* * *$ & 6 \\
\hline \multicolumn{3}{|c|}{ Topramezone } & & \\
\hline HPPD-SW & $3(0.5)$ & $15(2)$ & & - \\
\hline HPPD-RW & $8(1)$ & $72(10)$ & $* * *$ & 3 \\
\hline
\end{tabular}

a Abbreviations: HPPD-SW, 4-hydroxyphenylpyruvate dioxygenase (HPPD)-inhibiting herbicide-susceptible waterhemp biotype collected from a field in Clay County, NE in 2014; HPPD-RW, HPPD-inhibiting herbicide-resistant waterhemp biotype from a field in Platte County, NE in 2013.

${ }^{\mathrm{b}} \mathrm{ED}_{50}$, effective dose required to control $50 \%$ population; $\mathrm{ED}_{90}$, effective dose required to control $90 \%$ population.

${ }^{\mathrm{c}} \mathrm{HPPD}-\mathrm{RW}$ vs HPPD-SW $t$-statistics comparison of $\mathrm{ED}_{50}$, ${ }^{* * *} \alpha<0.01$.

${ }^{\mathrm{d}}$ Resistance level was calculated by dividing $\mathrm{ED}_{50}$ value of HPPD-RW by HPPD-SW for each herbicide.

The HPPD-RW is neither acetolactate synthase nor triazine resistant, and results of PRE-only herbicide programs suggest that PRE herbicide options are available for effective control of the HPPD-RW in corn.

POST-Only Herbicide Program. Four POST herbicide programs provided $\geq 90 \%$ control of the HPPD-RW at 21 DAT with $\geq 84 \%$ density reduction at 35 DAT (Table 8). For example, glyphosate (Treatment 1) provided $\geq 93 \%$ HPPD-RW control and density reduction. Thus, the HPPD-RW was very sensitive to glyphosate due to the fact that the experimental site had been under seed corn production at least for last five years with no use of glyphosate. Mesotrione/atrazine + diflufenzopyr/ dicamba (Treatment 2), mesotrione/atrazine + glufosinate (Treatment 3), and mesotrione/atrazine + metribuzin (Treatment 4) also provided $92 \%$ control 


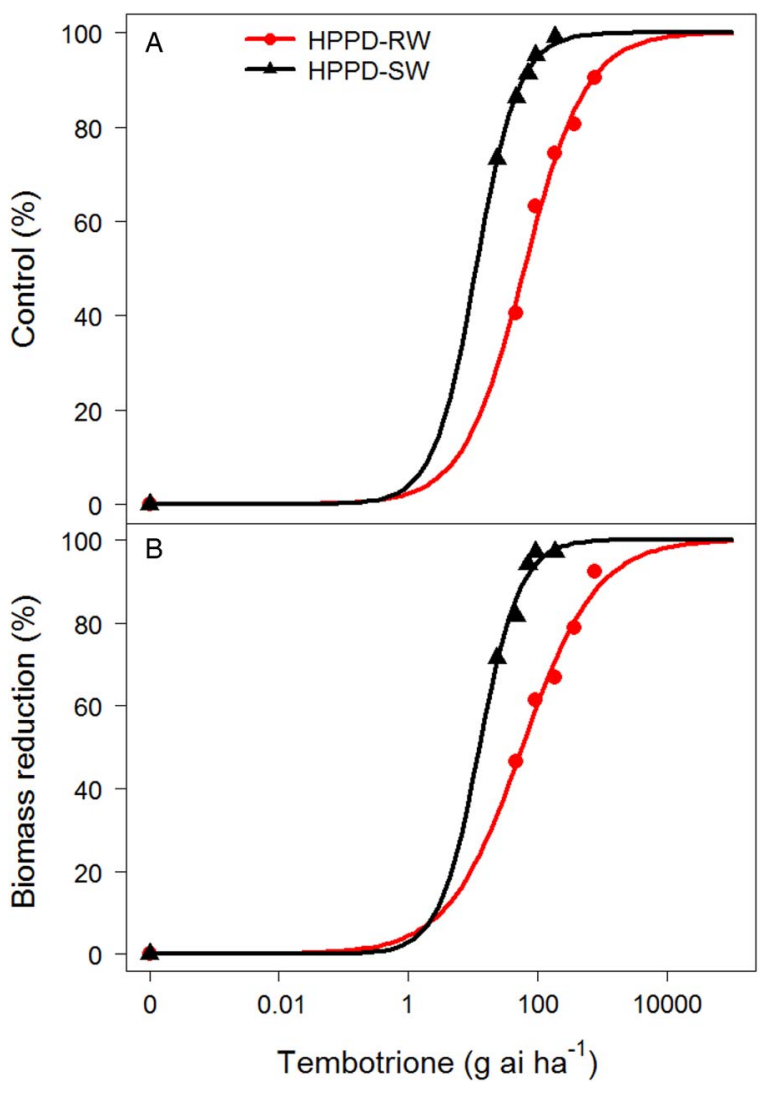

Figure 2. Control of (A) and biomass reduction (B) of 8- to 10-cm tall HPPD-inhibiting herbicide resistant (HPPD-RW) and susceptible (HPPD-SW) waterhemp biotype at $21 \mathrm{~d}$ after treatment with POST-applied tembotrione in dose-response studies under greenhouse conditions.

of HPPD-RW at 21 DAT. This is due to synergistic effect of HPPD-inhibiting herbicides and photosystem II-inhibiting herbicides (e.g., atrazine and metribuzin). Previous studies have confirmed improved control of Amaranthus species in corn by tank-mixing HPPDand photosystem II-inhibiting herbicides (Abendroth et al. 2006; Woodyard et al. 2009).

There was no difference in HPPD-RW control (21 DAT) between glyphosate and mesotrione/ atrazine in tank mixtures with diflufenzopyr/ dicamba, glufosinate, or metribuzin. All of these treatments resulted in $\geq 92 \%$ HPPD-RW control. Mesotrione/atrazine + metribuzin caused 15\% temporary stunting in corn at 10 DAT (data not shown). Fluthiacet-methyl + mesotrione showed poor (53\%) control of the HPPD-RW at 21 DAT (Table 8). Similar results were obtained by Jhala et al. (2014), who reported that fluthiacet-methyl used alone was not effective in controlling Amaranthus species.

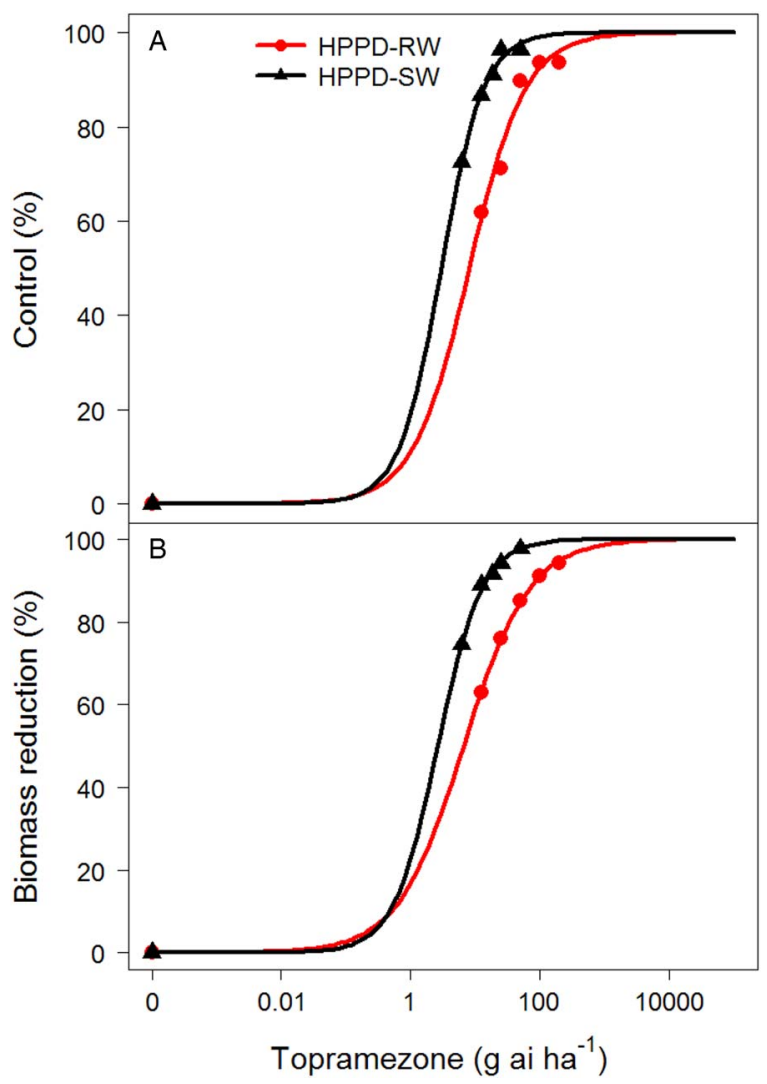

Figure 3. Control of (A) and biomass reduction (B) of 8- to 10-cm tall HPPD-inhibiting herbicide resistant (HPPD-RW) and susceptible (HPPD-SW) waterhemp biotype at $21 \mathrm{~d}$ after treatment with POST-applied topramezone in dose-response studies under greenhouse conditions.

The results of POST-only herbicide programs indicated that glyphosate, and premix of mesotrione/ atrazine tank mixed with synthetic auxins glufosinate and metribuzin, are effective herbicide programs for control of HPPD-RW in corn.

PRE $\mathrm{fb}$ POST Herbicide Programs. Most PRE $\mathrm{fb}$ POST herbicide programs provided $\geq 83 \%$ control and density reduction of HPPD-RW at 32 DAPOST (Table 9). The HPPD-RW was $\geq 86 \%$ controlled with PRE herbicide 30 DAPRE. The HPPD-RW control (\%) in PRE was higher when treated with $2,780 \mathrm{~g} \mathrm{ha}^{-1}$ (Treatments 9, 10, and 11) than $1,550 \mathrm{~g} \mathrm{ha}^{-1}$ (Treatment 8) of mesotrione/ $S$-metolachlor/atrazine. Furthermore, adding atrazine $\left(1,080 \mathrm{~g} \mathrm{ha}^{-1}\right)$ to mesotrione/ atrazine/S-metolachlor $\left(2,780 \mathrm{~g} \mathrm{ha}^{-1}\right)$ did not improve the HPPD-RW control (\%). The mesotrione/atrazine/ $S$-metolachlor $\left(2,780 \mathrm{~g} \mathrm{ha}^{-1}\right)$ provided nearly complete or complete control of HPPD-RW. This mixture, with 
Table 6. Estimated $\mathrm{ED}_{50}$ and $\mathrm{ED}_{90}$ values based on biomass reduction (\%) in 8- to 10-cm HPPD-inhibiting herbicideresistant (HPPD-R) and susceptible (HPPD-S) waterhemp biotype $21 \mathrm{~d}$ after treatment in a dose-response study with mesotrione, tembotrione, and topramezone, conducted under greenhouse conditions at the University of Nebraska-Lincoln.

\begin{tabular}{|c|c|c|c|c|}
\hline \multirow[b]{2}{*}{ Biotype $^{a}$} & \multicolumn{2}{|c|}{ HPPD-inhibiting herbicides ${ }^{b}$} & \multirow[b]{2}{*}{$P$-value ${ }^{\mathrm{c}}$} & \multirow[b]{2}{*}{ Resistance level $^{\mathrm{d}}$} \\
\hline & $\overline{\mathrm{ED}_{50}( \pm \mathrm{SE})}$ & $\mathrm{ED}_{90}( \pm \mathrm{SE})$ & & \\
\hline & 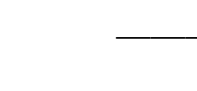 & 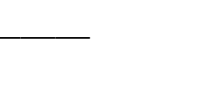 & & \\
\hline HPPD-SW & $20(1)$ & $123(10)$ & & \\
\hline HPPD-RW & $355(27)$ & - & $* * *$ & 18 \\
\hline HPPD-SW & $12(1)$ & $61(4)$ & & \\
\hline HPPD-RW & $56(4)$ & - & $* * *$ & 5 \\
\hline $\begin{array}{l}\text { HPPD-SW } \\
\text { HPPD-RW }\end{array}$ & $\begin{array}{l}3(0.4) \\
7(1)\end{array}$ & $\begin{array}{l}15(1) \\
86(13)\end{array}$ & $* * *$ & 2 \\
\hline
\end{tabular}

${ }^{a}$ Abbreviations: HPPD-SW, 4-hydroxyphenylpyruvate dioxygenase (HPPD)-inhibiting herbicide-susceptible waterhemp biotype collected from a field in Clay County, NE in 2014; HPPD-RW, HPPD-inhibiting herbicide-resistant waterhemp biotype from a field in Platte County, NE in 2013.

${ }^{\mathrm{b}} \mathrm{ED}_{50}$, effective dose required to control $50 \%$ population; $\mathrm{ED}_{90}$, effective dose required to control $90 \%$ population.

${ }^{\mathrm{c}} \mathrm{HPPD}-\mathrm{RW}$ vs HPPD-SW $t$-statistics comparison of $\mathrm{ED}_{50},{ }^{* * *} \alpha<0.01$.

${ }^{\mathrm{d}}$ Resistance level was calculated by dividing $\mathrm{ED}_{50}$ value of HPPD-RW by HPPD-SW for each herbicide.

or without atrazine, resulted in $\geq 97 \%$ HPPD-RW control at 30 DAPRE. Moreover, acetochlor/flumetsulam/clopyralid, pyroxasulfone/fluthiacet-methyl/atrazine, saflufenacil/dimethenamid- $P+\operatorname{dimethenamid}-P$, and thiencarbazone-methyl/isoxaflutole + atrazine provided $\geq 95 \%$ HPPD-RW control.

Treatments $\mathrm{fb}$ POST application of glyphosate alone (Treatments 3, 6, 9, and 13) or glyphosate +

Table 7. Effect of PRE-only herbicide programs on HPPD-inhibiting resistant waterhemp control (\%) and population density reduction (\%) in field experiments conducted in 2013 and 2014 near Columbus, NE.

\begin{tabular}{|c|c|c|c|c|c|c|}
\hline & \multirow{2}{*}{$\begin{array}{l}\text { HPPD-inhibiting herbicide-resistant } \\
\text { waterhemp }{ }^{\text {a }}\end{array}$} & \multirow[b]{3}{*}{ Rate } & \multicolumn{3}{|c|}{ Control $^{\mathrm{b}}$} & Density reduction $^{b}$ \\
\hline & & & \multicolumn{4}{|c|}{$\mathrm{DAT}^{\mathrm{a}}$} \\
\hline \multirow[t]{2}{*}{ Treatment } & Herbicide $^{c}$ & & 30 & 41 & 56 & 56 \\
\hline & & $\mathrm{g}$ ai ha ${ }^{-1}$ & & & & \\
\hline 1 & Acetochlor/flumetsulam/clopyralid & 1,490 & $93 \mathrm{bc}$ & $85 \mathrm{~d}$ & $85 \mathrm{bc}$ & $91 \mathrm{bcd}$ \\
\hline 2 & Mesotrione/atrazine/S-metolachlor + acetochlor & $2,780+1,470$ & $97 \mathrm{a}$ & $98 \mathrm{a}$ & 96 a & $98 \mathrm{a}$ \\
\hline 5 & Pyroxasulfone & 270 & $95 \mathrm{abc}$ & $94 \mathrm{abc}$ & $92 \mathrm{ab}$ & $95 \mathrm{abc}$ \\
\hline 6 & Pyroxasulfone/fluthiacet-methyl/atrazine & 1,260 & $96 \mathrm{ab}$ & $94 \mathrm{abc}$ & $92 \mathrm{ab}$ & $88 \mathrm{~cd}$ \\
\hline 7 & Pyroxasulfone + saflufenacil + atrazine & $149+75+560$ & $96 \mathrm{ab}$ & $97 \mathrm{ab}$ & $93 \mathrm{ab}$ & $96 \mathrm{ab}$ \\
\hline 8 & Suflafenacil/dimethenamid- $P+$ dimethenamid- $P$ & $730+263$ & $96 \mathrm{ab}$ & $91 \mathrm{bcd}$ & $86 \mathrm{bc}$ & $84 \mathrm{~d}$ \\
\hline 9 & Thiencarbazone-methyl/isoxaflutole + atrazine & $129+1,800$ & $90 \mathrm{~cd}$ & $91 \mathrm{bcd}$ & $75 \mathrm{c}$ & $60 \mathrm{e}$ \\
\hline
\end{tabular}

${ }^{a}$ Abbreviations: DAT, d after treatment; HPPD, 4-hydroxyphenylpyruvate dioxygenase. The control (0\%) data of non-treated experimental unit were not included in analysis. Density reduction (\%) was calculated on the basis of comparison with density (plants $\mathrm{m}^{2}$ ) of non-treated experimental unit.

${ }^{\mathrm{b}}$ Means presented within each column with no common letter(s) are significantly different according to Fisher's Protected LSD test where $P \leq 0.05$.

${ }^{\mathrm{c}}$ Herbicide premix (/); herbicide tankmix $(+)$.

${ }^{\mathrm{d}}$ ANOVA, ${ }^{* * *} \alpha<0.01$. 
Table 8. Effect of POST-only herbicide programs on HPPD-inhibiting herbicide-resistant-waterhemp control (\%) and population density reduction (\%) in field experiments conducted in 2013 and 2014 near Columbus, NE.

\begin{tabular}{|c|c|c|c|c|c|c|}
\hline & & & \multicolumn{3}{|c|}{ Control $^{\mathrm{b}}$} & Density reduction $^{\mathrm{b}}$ \\
\hline \multicolumn{2}{|c|}{ HPPD-inhibiting herbicide-resistant waterhemp ${ }^{a}$} & \multirow[b]{2}{*}{ Rate } & \multicolumn{4}{|c|}{$\mathrm{DAT}^{\mathrm{a}}$} \\
\hline Treatment & Herbicide $^{c}$ & & 7 & 14 & 21 & 35 \\
\hline 1 & & $\begin{array}{l}\mathrm{g} \text { ai (ae) ha } \mathrm{ha}^{-1} \\
1.320\end{array}$ & 987 & 98 & $-\%$ & \\
\hline $\begin{array}{l}1 \\
2\end{array}$ & Mesotrione/atrazine + diflufenzopyr/ dicamba & $\begin{array}{c}1,30 \\
650+196\end{array}$ & $81 \mathrm{c}$ & $87 \mathrm{~b}$ & $92 \mathrm{a}$ & $91 \mathrm{ab}$ \\
\hline 3 & Mesotrione/atrazine + glufosinate & $650+595$ & $98 \mathrm{a}$ & $96 \mathrm{a}$ & $92 \mathrm{a}$ & $84 \mathrm{~b}$ \\
\hline 4 & Mesotrione/atrazine + metribuzin & $650+210$ & $94 \mathrm{~b}$ & $91 \mathrm{~b}$ & $92 \mathrm{a}$ & $97 \mathrm{a}$ \\
\hline & Mesotrione + fluthiacet-methyl & $105+7$ & $82 \mathrm{c}$ & $54 \mathrm{c}$ & $53 \mathrm{~b}$ & $65 c$ \\
\hline$p$-value ${ }^{\mathrm{d}}$ & & & *** & $* * *$ & $* * *$ & $* * *$ \\
\hline
\end{tabular}

${ }^{a}$ Abbreviations: DAT, $\mathrm{d}$ after treatment; HPPD, 4-hydroxyphenylpyruvate dioxygenase. The control (\%) data of non-treated experimental unit were not included in analyses. Density reduction (\%) was calculated on the basis of comparison with density (plants $\mathrm{m}^{-2}$ ) of non-treated experimental unit.

${ }^{\mathrm{b}}$ Means presented within each column with no common letter(s) are significantly different according to Fisher's Protected LSD test where $P \leq 0.05$.

${ }^{\mathrm{c}}$ Herbicide premix (/); herbicide tankmix $(+)$.

${ }^{\mathrm{d}}$ ANOVA, ${ }^{* * *} \alpha<0.01$.

topramezone + atrazine (Treatment 1), glyphosate + diflufenzopyr/dicamba (Treatment 5), and glyphosate/S-metolachlor/mesotrione + atrazine (Treatment 8) provided $\geq 95 \%$ HPPD-RW control and density reduction at 32 DAPOST. Diflufenzopyr/ dicamba resulted in $86 \%$ to $91 \%$ control of HPPD-RW, but the control was improved to $97 \%$ when glyphosate was tank-mixed with diflufenzopyr/ dicamba (Treatment 5). Non-glyphosate treatments, including topramezone + diflufenzopyr/dicamba + atrazine (Treatment 2), diflufenzopyr/dicamba + atrazine (Treatment 4), atrazine $+S$-metolachlor + glufosinate (Treatment 7) and topramezone + diflufenzopyr/ dicamba (Treatment 14) resulted in $\geq 94 \%$ HPPDRW control and density reduction at 32 DAPOST. These results suggest that many herbicide options are available to manage HPPD-RW in corn, at least in Nebraska and the upper Midwest.

Herbicide rotations and/or mixtures of active ingredients that have different SOA have been recommended by researchers as a way to prevent or delay the evolution of resistant weeds (Beckie 2006; Gressel and Segel 1990; Norsworthy et al. 2012; Wrubel and Gressel 1994). Similarly, Livingston et al. (2015) suggested that the lowest risk of evolving herbicide resistance occurred when both PRE and POST herbicide applications are part of a systematic approach to weed control. In addition, the sequential application of PRE $\mathrm{fb}$ POST would also help in fields with substantial waterhemp density, which has tendency to emerge over a longer period of time (Cordes et al. 2004; Schuster and Smeda 2007).

This study confirmed the first case of HPPD-RW in Nebraska, and the third in the United States (Heap 2016b). This biotype showed the highest resistance to mesotrione, followed by tembotrione and topramezone, most likely due to the longer history of mesotrione use at the study site in a continuous seed corn production system. The results indicate that there are herbicide programs that have the potential to provide effective control of HPPD-RW in corn. Tactics for minimizing the risk of herbicide resistance should be based on the principles of integrated weed management, especially utilizing mixtures or premixes of herbicides with different SOA. Despite availability of alternative herbicides, the spread of HPPD-inhibiting herbicide resistance in Amaranthus spp. is increasing across other parts of Nebraska and the United States (Hausman et al. 2011; Jhala et al. 2014; McMullan and Green 2011; Thompson et al. 2012), which is of great concern because it limits the effectiveness of mesotrione, tembotrione, and topramezone on pigweed species. Future research is needed to confirm the mechanism of resistance to HPPDinhibiting herbicides observed in this biotype from Nebraska. 
Table 9. Effect of PRE followed by POST herbicide programs on HPPD-inhibiting herbicide-resistant-waterhemp control (\%) and population density reduction (\%) in field experiments conducted in 2013 and 2014 near Columbus, NE.

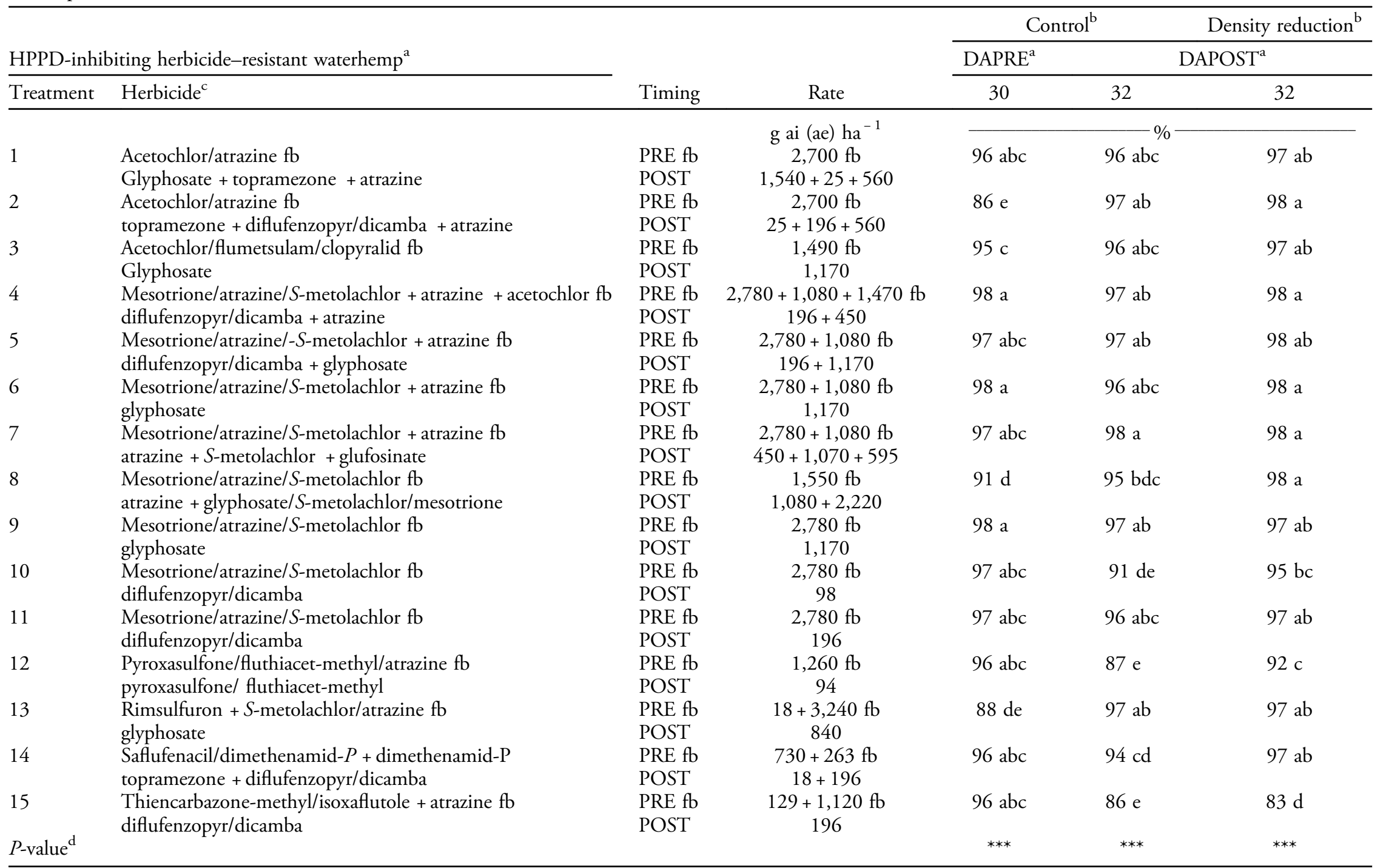

${ }^{a}$ Abbreviations: DAPRE, d after PRE; DAPOST, d after POST; HPPD, 4-hydroxyphenylpyruvate dioxygenase. The control (\%) data of non-treated experimental unit were not included in analyses. Density reduction (\%) was calculated on the basis of comparison with density (plants $\mathrm{m}^{-2}$ ) of non-treated experimental unit.

${ }^{\mathrm{b}}$ Means presented within each column with no common letter(s) are significantly different according to Fisher's Protected LSD test where P $\leq 0.05$.

${ }^{\mathrm{c}}$ Herbicide premix (/); herbicide tankmix (+); fb, followed by.

${ }^{\mathrm{d}}$ ANOVA, ${ }^{* * *} \alpha<0.01$ 


\section{Acknowledgments}

The authors thank CAPES (Brazilian Government Foundation) - Proc. $\mathrm{n}^{\circ}$ 9112-13-8, for financial support to the graduate student involved in this study. We appreciate the help of Sergio Oliveira, Kyle Kardell, and Amanda Winstead in this project.

\section{Literature Cited}

Abendroth JA, Martin AR, Roeth FW (2006) Plant response to combinations of mesotrione and photosystem II Inhibitors. Weed Technol 20:267-274

Beckie HJ (2006) Herbicide-resistant weeds: management tactics and practices. Weed Technol 20:793-814

Beckie HJ, Reboud X (2009) Selecting for weed resistance: herbicide rotation and mixture. Weed Technol 23:363-370

Bernards ML, Crespo RJ, Kruger GR, Gaussoin R, Tranel PJ (2012) A waterhemp (Amaranthus tuberculatus) population resistant to 2,4-D. Weed Sci 60:379-384

Bollman JD, Boerboom CM, Becker RL, Fritz VA (2008) Efficacy and tolerance to HPPD-inhibiting herbicides in sweet corn. Weed Technol 22:666-674

Butts TR, Norsworthy JK, Kruger GR, Sandell LD, Young BG, Steckel LE, Loux MM, Bradley KW, Conley SP, Stoltenberg DE, Arriaga FJ, Davis VM (2016) Management of pigweed (Amaranthus spp.) in glufosinate-resistant soybean in the midwest and mid-south. Weed Technol 30:355-365

Conover WJ, Johnson ME, Johnson MM (1981) A comparative study of tests for homogeneity of variances, with applications to the outer continental shelf bidding data. Technometrics 23:351-361

Cordes JC, Johnson WG, Scharf P, Smeda RJ (2004) Lateemerging common waterhemp (Amaranthus rudis) interference in conventional tillage corn. Weed Technol 18:999-1005

Culpepper AS (2006) Glyphosate-induced weed shifts. Weed Technol 20:277-281

Duke SO (2012) Why have no new herbicide modes of action appeared in recent years? Pest Manag Sci 68:505-512

Felix J, Owen MD (1999) Weed population dynamics in land removed from the conservation reserve program. Weed Sci 47:511-517

Gitsopoulos TK, Melidis V, Evgenidis G (2010) Response of maize (Zea mays L.) to post-emergence applications of topramezone. Crop Prot 29:1091-1093

Gressel J, Segel LA (1990) Modelling the effectiveness of herbicide rotations and mixtures as strategies to delay or preclude resistance. Weed Technol 4:186-198

Grossmann K, Ehrhardt T (2007) On the mechanism of action and selectivity of the corn herbicide topramezone: a new inhibitor of 4-hydroxyphenylpyruvate dioxygenase. Pest Manag Sci 63:429-439

Hager AG, Wax LM, Stoller EW, Bollero GA (2002) Common waterhemp (Amaranthus rudis) interference in soybean. Weed Technol 50:607-610

Hartzler R, Buhler D, Stoltenberg D (1999) Emergence characteristics of four annual weed species. Weed Sci 47:578-584
Hausman NE, Singh S, Tranel PJ, Riechers DE, Kaundun SS, Polge ND, Thomas DA, Hager AG (2011) Resistance to HPPD-inhibiting herbicides in a population of waterhemp (Amaranthus tuberculatus) from Illinois, United States. Pest Manag Sci 67:258-261

Hausman N, Tranel P, Riechers D, Hager AG (2015) Responses of a waterhemp (Amaranthus tuberculatus) population resistant to HPPD-inhibiting herbicides to foliar-applied herbicides. Weed Technol 30:106-115

Hausman NE, Tranel PJ, Riechers DE, Maxwell DJ, Gonzini LC, Hager AG (2013) Responses of an HPPD inhibitor-resistant waterhemp (Amaranthus tuberculatus) population to soilresidual herbicides. Weed Technol 27:704-711

Heap I (2016a). The International Survey of Herbicide Resistant Weeds. Weeds Resistant Tall Waterhemp Globally. http:// weedscience.org/Summary/Species.aspx. Accessed April 05, 2016

Heap I (2016b). The International Survey of Herbicide Resistant Weeds. Weeds Resistant to HPPD-inhibitors. http://weedscience. org/Summary/MOA.aspx. Accessed April 02, 2016

Jhala AJ, Sandell LD, Rana N, Kruger GR, Knezevic SZ (2014) Confirmation and control of triazine and 4-hydroxyphenylpyruvate dioxygenase-inhibiting herbicide-resistant Palmer amaranth (Amaranthus palmeri) in Nebraska. Weed Technol 28:28-38

Johnson G, Breitenbach F, Behnken L, Miller R, Hoverstad T, Gunsolus J (2012) Comparison of herbicide tactics to minimize species shifts and selection pressure in glyphosate-resistant soybean. Weed Technol 26:189-194

Knezevic SZ, Streibig JC, Ritz C (2007) Utilizing R software package for dose-response studies: the concept and data analysis. Weed Technol 21:840-848

Kumar V, Jha P (2015) Effective preemergence and postemergence herbicide programs for kochia control. Weed Technol 29:24-34

Livingston M, Fernandez-Cornejo J, Unger J, Osteen C, Schimmelpfennig D, Park T, Lambert D (2015) The Economics of Glyphosate Resistance Management in Corn and Soybean Production, Washington, DC: US Department of Agriculture ERR. 184:45 p

Loux MM, Dobbels AF, Johnson WG, Young BG (2011) Effect of residual herbicide and postemergence application timing on weed control and yield in glyphosate-resistant corn. Weed Technol 25:19-24

Mahoney KJ, Shropshire C, Sikkema PH (2014) Weed management in conventional- and no-till soybean using flumioxazin/ pyroxasulfone. Weed Technol 28:298-306

McMullan PM, Green JM (2011) Identification of a tall waterhemp (Amaranthus tuberculatus) biotype resistant to HPPD-inhibiting herbicides, atrazine, and thifensulfuron in Iowa. Weed Technol 25:514-518

Mitchell G, Bartlett DW, Fraser TEM, Hawkes TR, Holt DC, Townson JK, Wichert RA (2001) Mesotrione: a new selective herbicide for use in maize. Pest Manag Sci 57:120-128

Norsworthy JK, Ward SM, Shaw DR, Llewellyn RS, Nichols RL, Webster TM, Bradley KW, Frisvold G, Powles SB, Burgos NR (2012) Reducing the risks of herbicide resistance: best management practices and recommendations. Weed Sci 60(sp1), 31-62

Nurse RE, Hamill AS, Swanton CJ, Tardif FJ, Sikkema PH (2010) Weed control and yield response to mesotrione in maize (Zea mays). Crop Protect 29:652-657 
Prince JM, Shaw DR, Givens WA, Owen MD, Weller SC, Young BG, Wilson RG, Jordan DL (2012) Benchmark study: I. Introduction, weed population, and management trends from the benchmark survey 2010. Weed Technol 26:525-530

Ritz C, Streibig JC (2005) Bioassay analysis using R. J of Stat Soft 12:1-22

Sarangi D, Sandell LD, Knezevic SZ, Aulakh JS, Lindquist JL, Irmak S, Jhala AJ (2015) Confirmation and control of glyphosate-resistant common waterhemp (Amaranthus rudis) in Nebraska. Weed Technol 29:82-92

Sauer JD (1967) The grain amaranths and their relatives: a revised taxonomic and geographic survey. Ann Mo Bot Gard 54: 103-137

Schuster CL, Smeda RJ (2007) Management of Amaranthus rudis $S$. in glyphosate-resistant corn (Zea mays L.) and soybean (Glycine max L. Merr.). Crop Prot 26:1436-1443

Steckel LE, Sprague CL (2004) Common waterhemp (Amaranthus rudis) interference in corn. Weed Sci 52:359-364

Sutton P, Richards C, Buren L, Glasgow L (2002) Activity of mesotrione on resistant weeds in maize. Pest Manag Sci 58:981-984

Thompson CR, Peterson D, Lally NG (2012). Characterization of HPPD-resistant Palmer amaranth. Proceedings of the $52^{\text {nd }}$ Annual Conference of Weed Science Society of America. Waikoloa, Hawaii. http://wssaabstracts.com/public/9/proceedings. html. Accessed December 23, 2016

Tranel PJ, Riggins CW, Bell MS, Hager AG (2011) Herbicide resistances in Amaranthus tuberculatus: a call for new options. J Agric Food Chem 59:5808-5812

Waselkov KE, Olsen KM (2014) Population genetics and origin of the native North American agricultural weed waterhemp (Amaranthus tuberculatus; Amaranthaceae). Am J Bot 101: 1726-1736

Woodyard AJ, Bollero GA, Riechers DE (2009) Broadleaf weed management in corn utilizing synergistic postemergence herbicide combinations. Weed Technol 23:513-518

Wrubel RP, Gressel J (1994) Are herbicide mixtures useful for delaying the rapid evolution of resistance? A case study. Weed Technol 8:635-648

Received June 27, 2016, and approved October 17, 2016.

Associate Editor for this paper: William Johnson, Purdue University. 